Ciência Florestal, Santa Maria, v. 23, n. 4, p. 643-654, out.-dez., 2013

ISSN 0103-9954

\title{
MÉTODOS DE ESTRATIFICAÇÃO VERTICAL EM FLORESTA ESTACIONAL SEMIDECIDUAL
}

\author{
METHODS OF VERTICAL STRATIFICATION IN SEMIDECIDUOUS SEASONAL FOREST
}

\author{
Rafaella De Angeli Curto ${ }^{1}$ Gilson Fernandes da Silva ${ }^{2}$ José Eduardo Macedo Pezzopane ${ }^{3}$ \\ José Franklim Chichorro ${ }^{4}$ Rômulo Môra ${ }^{5}$
}

\begin{abstract}
RESUMO
O presente estudo foi realizado em um fragmento de Floresta Estacional Semidecidual de 52 ha, conhecido como Floresta do Rosal, no município de Guaçuí-ES e teve como objetivo testar metodologias de estratificação vertical, avaliando a adequação dessas metodologias baseada na classificação sucessional ecológica das espécies. Para tanto, empregou-se o método de amostragem de área fixa, sendo distribuídas 16 parcelas de $600 \mathrm{~m}^{2}$, de forma sistemática no campo. Todos os indivíduos com $D A P \geq 5 \mathrm{~cm}$ tiveram suas alturas totais medidas de forma direta. As árvores passaram por processo de identificação botânica e classificação quanto ao grupo ecológico. A análise da estrutura vertical de espécies arbóreas foi feita pela distribuição do número de árvores nos estratos, utilizando-se quatro métodos: Método 1 - Souza (1990), Método 2 - Souza et al. (2003), Método 3 - IUFRO, e Método 4 - Calegário et al. (1994). Os resultados das classificações obtidas foram comparados à classificação sucessional das espécies, de acordo com os grupos ecológicos a que pertencem. O Método 1 não permitiu análise detalhada sobre o comportamento das espécies no estrato II por apresentar tendências fortes em concentrar um maior número de indivíduos no referido estrato, já o Método 2 permitiu um maior detalhamento. O Método 3 minimizou o problema encontrado no Método 1, porém, a mudança da altura dominante da amostragem pode mudar toda a discussão, demonstrando fragilidade do método. O Método 4 não trouxe bons resultados para a estratificação da floresta em estudo, pois a dividiu em apenas dois estratos de altura total.
\end{abstract}

Palavras-chave: altura total; classificação sucessional; estratos arbóreos.

\section{ABSTRACT}

This study was conducted in a semideciduous forest fragment of 52 hectares, known as Rosal Forest, in the municipality of Guaçuí, Espírito Santo state. It had the objective to test vertical stratification methodologies, evaluating the appropriateness of these methodologies based on the ecological successional classification of the species. To do so, the method of sampling fixed area was used, and distributed 16 plots of $600 \mathrm{~m}^{2}$, in a systematic way. All individuals with $\mathrm{DBH} \geq 5 \mathrm{~cm}$, had their total height measures directly. The trees went through the botanical classification identification process and had their classifications in accordance with their ecological groups. The analysis of the tree vertical structure was made by the distribution of the

1 Engenheira Florestal, MSc., Doutoranda em Engenharia Florestal da Universidade Federal do Paraná, Av. Pref. Lothário Meissner, 900, Jardim Botânico, Campus III, CEP 80210-170, Curitiba (PR), Brasil. rafaellacurto@yahoo.com.br

2 Engenheiro Florestal, Dr., Professor Associado I do Departamento de Engenharia Florestal, Universidade Federal do Espírito Santo, Campus Universitário de Alegre, Alto Universitário, Caixa Postal 16, CEP 29500-000, Alegre (ES), Brasil. fernandes5012@gmail.com

3 Engenheiro Florestal, Dr., Professor Associado I do Departamento de Engenharia Florestal, Universidade Federal do Espírito Santo, Campus Universitário de Alegre, Alto Universitário, Caixa Postal 16, CEP 29500-000, Alegre (ES), Brasil. pezzopane2007@yahoo.com.br

4 Engenheiro Florestal, Dr., Professor Associado do Departamento de Engenharia Florestal, Universidade Federal do Espírito Santo, Campus Universitário de Alegre, Alto Universitário, Caixa Postal 16, CEP 29500-000, Alegre (ES), Brasil. jfufes@gmail.com

5 Engenheiro Florestal, MSc., Professor Assistente I do Departamento de Engenharia Florestal, Faculdade de Engenharia Florestal, Universidade Federal de Mato Grosso, Av. Fernando Côrrea da Costa , s/n, Boa Esperança, CEP 78060-900, Cuiabá (MT), Brasil.romulomef@yahoo.com.br

Recebido para publicação em 18/11/2011 e aceito em 12/06/2012 
number of trees in the strata, using four methods: Method 1 - Souza (1990), Method 2 - Souza et al. (2003), Method 3 - IUFRO, and Method 4 - Calegário et al. (1994). The classification results were compared to the successional classification of species, according to the ecologic groups that they belong to. The method 1 did not allow a detailed analysis about the behavior of stratum II species, because of the strong tendencies to concentrate a greater number of individuals in this stratum, already the Method 2 has allowed a greater detail. The Method 3 minimized the problem encountered in Method 1, but the dominant height change of the sample can change the whole discussion, demonstrating the method weakness. Method 4 did not bring good results for the forest stratification under study, because this method only divided in two height strata.

Keywords: total height; successional classification; tree layers strata.

\section{INTRODUÇÃO}

Segundo Durigan (2009), a maioria dos estudos que trata da caracterização de comunidades vegetais no Brasil compreende estudos fitossociológicos baseados em tabelas de Valor de Importância (VI) e de Valor de Cobertura (VC) das espécies. Contudo, para uma caracterização real da ordem de importância ecológica das espécies arbóreas em uma floresta, somente indicadores da estrutura horizontal não são suficientes. Assim, a inclusão da estrutura vertical na análise estrutural das florestas é de grande importância.

A importância da avaliação de espécies nos diferentes estratos da floresta é devida ao fato de cada espécie ter seu lugar assegurado na estrutura e composição, quando se encontra representada em todos os estratos, excetuando-se a essa regra àquelas que nunca chegam a passar do piso inferior, por características próprias, sendo pouco desenvolvidas e muito tolerantes à sombra (FINOL, 1975).

Através da avaliação da estrutura vertical em populações, pode-se identificar o comportamento ecológico e o hábito de cada população, essa análise prevê informações importantes para a compreensão das características de cada espécie, dando embasamento para entendimento das estratégias de regeneração natural, crescimento e sobrevivência (SANQUETTA, 1995). Porém, apesar da importância e de a estrutura horizontal ser bem caracterizada em estudos fitossociológicos, a estrutura vertical é pouco explorada, sobretudo pela dificuldade em analisá-la.

Para estratificar florestas inequiâneas, em termos de altura total, alguns métodos têm sido propostos e empregados. Esses métodos são citados por autores como Longhi (1980), que divide em três partes iguais a frequência relativa das alturas encontradas na floresta; classificação da IUFRO (LAMPRECHT, 1990), a qual parte da altura dominante $\left(h_{d o m}\right)$ da floresta para distinguir os estratos;
Souza (1990), que estratifica a floresta em três estratos de altura total com base na média e no desvio padrão; Calegário et al. (1994), em que a estratificação é feita com base no teste de qui-quadrado; Sanquetta (1995), que propõe a estratificação denominada de diagrama h-M; e Souza et al. (2003), que empregaram análise multivariada para estratificação.

De acordo com Durigan (2009), não há um método reconhecido para se determinar o número de estratos que compõem o dossel florestal, de modo que tentativas de estratificação em florestas tropicais, em geral, têm sido baseadas na divisão arbitrária das árvores em classes de altura. Apesar da disponibilidade de vários métodos, ainda que importante para a compreensão da estrutura de uma floresta, a diferenciação segura dos estratos em uma comunidade florestal, com base estatística, é um desafio a ser vencido.

Durigan (2009) também comenta que classificação sucessional, ainda que imprecisa, pode ser útil na compreensão da estrutura e dinâmica de comunidades florestais, desde que utilizada com precauções, nunca como se fosse um atributo intrínseco das espécies. A classificação sucessional das espécies tem forte componente subjetivo e, apesar dos esforços dos pesquisadores, muitas espécies não se enquadram nos moldes dos chamados grupos ecológicos ou grupos sucessionais.

Brown (1919) apud Durigan et al. (2000) constatou que a estratificação vertical é complexa, com indivíduos jovens do estrato superior ocorrendo nos estratos inferiores e entre os estratos. Assim, a identificação de estratos verticais na floresta é sempre controvertida e, em florestas tropicais, nem sempre a separação entre eles é perceptível.

Apesar disso, a avaliação da estrutura vertical em florestas inequiâneas é importante, uma vez que pode ser de grande valor para a sustentabilidade do manejo. Assim, a tarefa de reconhecer estratos de árvores na floresta é um dos principais elementos do estudo da estrutura vertical (SANQUETTA, 1995). 
Rodrigues (1995) cita que espécies pioneiras têm função cicatrizadora de ambientes perturbados, e no outro extremo das pioneiras, têm-se as clímax, aqui denominadas de não pioneiras, que são as espécies finais na substituição sequencial de espécies na sucessão. Entre os dois extremos existe um grande número de espécies com características ou adaptações ecológicas intermediárias, sendo que quando as características são mais parecidas com as pioneiras são chamadas de secundárias iniciais e quando apresentam características mais próximas das espécies não pioneiras, são denominadas secundárias tardias.

Embora alguma estratificação seja evidente no dossel de florestas, ela não pode ser considerada como regra. Ainda assim, os extremos nas condições físicas, encontradas na interface entre a floresta e a atmosfera, justificam o reconhecimento dessa porção superior da floresta como estrato distinto. Várias características abióticas e bióticas da porção superior do dossel de florestas tropicais são diferentes daquelas das camadas inferiores da floresta. Por exemplo, a disponibilidade de luz e os níveis de raio ultraviloleta são notadamente maiores no dossel do que no sub-bosque (BASSET et al., 2003).

Considerando o exposto, teve-se como objetivo revisar e testar metodologias de estratificação da estrutura vertical de uma floresta estacional semidecidual, avaliando a adequação dessas metodologias com relação à classificação quanto ao grupo ecológico pertencente.

\section{METODOLOGIA}

\section{Caracterização da área de estudo}

O presente estudo foi desenvolvido em um fragmento de 52,04 hectares, coberto por Floresta Estacional Semidecidual, conhecido como Floresta do Rosal, pertencente à Usina Hidrelétrica Rosal, localizada no município de Guaçuí-ES. As coordenadas geográficas de acesso à entrada são $20^{\circ} 53^{\prime}$ latitude Sul e $41^{\circ} 42^{\prime}$ de longitude Oeste (SANSEVERO et al., 2006).

\section{Coleta de dados}

Foi realizado um inventário florestal da Floresta do Rosal por meio do método de amostragem de área fixa, sendo distribuídas 16 parcelas de 20 X $30 \mathrm{~m}\left(600 \mathrm{~m}^{2}\right)$, de forma sistemática no campo (com uma distância de 150 metros entre parcelas na linha e 200 metros entre linhas), em toda a extensão da floresta, totalizando uma área amostrada de 0,96 hectares. Em locais que apresentaram declividade de terreno foram precedidas as devidas correções, através de uma relação de cosseno, para que todas as parcelas tivessem áreas iguais, assim como mostrado a seguir (SOARES et al., 2006):

$$
\cos (\theta)=\frac{d}{d_{I}} \rightarrow d=d_{I} \cos (\theta) \rightarrow d_{I}=\frac{d}{\cos (\theta)}
$$

em que: $\theta$ a angulo de inclinação do terreno, em graus; $d_{I}=$ distância inclinada e; $d=$ distância no plano horizontal

Após distribuição das parcelas definidas no inventário florestal, todos os indivíduos com DAPs (diâmetro a altura do peito, igual a 1,30 m de altura do solo) igual ou superior a $5 \mathrm{~cm}$ foram identificados com plaquetas numeradas e presas aos fustes com arame.

Em todos os indivíduos identificados com plaquetas, foram medidas as respectivas alturas totais com régua telescópica de 15 metros de altura, e nas árvores com alturas maiores que 15 metros, foi realizada a escalada de todos os indivíduos a fim de obter sua altura real.

Depois de medidas e identificadas com plaquetas, as árvores passaram por um processo de identificação botânica. As amostras botânicas, tanto em estado reprodutivo quanto vegetativo, foram coletadas e acondicionadas em jornais, papelões e sacos plásticos. Posteriormente, o material foi prensado e seco em estufa.

Após a secagem, os materiais botânicos foram identificados em níveis de família, gênero e espécie com ajuda de bibliografia especializada e comparações com os materiais contidos na coleção do Herbário da Reserva Natural da Vale do Rio Doce, Linhares-ES. Em alguns casos, as amostras botânicas foram enviadas aos especialistas para identificação. Quando não foi possível a identificação, a amostra foi considerada como espécie não identificada.

Os exemplares coletados foram depositados no herbário do Departamento de Engenharia Florestal (DEF), do Centro de Ciências Agrárias da Universidade Federal do Espírito Santo (CCAUFES), em Jerônimo Monteiro-ES.

Foi utilizado o sistema Angiosperm Phylogeny Group III - APG III - para classificação das famílias (CHASE, 2009). Todos os nomes das espécies e seus respectivos autores foram confirma- 
dos e atualizados pelo site da Lista de Espécies da Flora do Brasil (2010).

\section{Classificação ecológica das espécies arbustivo- arbóreas}

A classificação de espécies em grupos ecológicos possibilita o manuseio de forma simplificada de um grande número de espécies, sendo agrupadas de acordo com as suas exigências e funções semelhantes.

Assim, para a classificação ecológica das espécies amostradas, foram realizadas classificações em duas categorias sucessionais, sendo elas: pioneiras e não pioneiras, com base nos critérios adotados por Rolim et al. (1999); Silva et al. (2003); Rolim (2006); Carvalho et al. (2007); Archanjo (2008); Moreira (2009); Dias Neto et al. (2009) e Pereira et al. (2010). Esses trabalhos foram utilizados como base para a classificação ecológica das espécies, por terem sido realizados em florestas estacionais semideciduais e em regiões próximas à área de estudo, nos estados de Minas Gerais e Espírito Santo, em que as espécies classificadas como secundárias iniciais foram determinadas como pioneiras, e as espécies classificadas como secundárias tardias, estabeleceu-se ser não pioneiras.

\section{Estrutura vertical}

A partir dos dados obtidos no inventário, a análise da estrutura vertical das espécies arbóreas foi feita pela distribuição do número de árvores nos diferentes estratos, por meio dos seguintes métodos:

\section{Método 1 - Souza (1990)}

Este método estratifica a floresta em três estratos de altura total com base na média e no desvio padrão, conforme Souza et al. (1998):

Estrato inferior: árvores com $h_{h}<(\bar{h}-s)$; $(\bar{h}+s)$; $\mathrm{e}$

Estrato médio: árvores com $(\bar{h}-s) \leq h_{1}<$

Estrato superior: árvores com $h_{1} \geq(\bar{h}+s)$.

em que: $\vec{h}=$ Média das alturas totais $\left(h_{l}\right)$ dos indivíduos amostrados e; $s=$ Desvio padrão das alturas totais $\left(h_{1}\right)$ dos indivíduos amostrados.

Os cálculos relativos a este método foram realizados no software Mata Nativa 3.0 (CIENTEC, 2010).
Método 2 - Souza et al. (2003)

Essa metodologia emprega a análise multivariada para estratificação. Para analisar a estrutura vertical, foram selecionados os dados de alturas totais de árvores amostradas com $D A P$ igual ou maior que $5,0 \mathrm{~cm}$.

As árvores individuais foram organizadas em ordem crescente de altura total, classificadas em classes com amplitude de $1 \mathrm{~m}$, e em seguida foi elaborada uma matriz $\mathrm{X}$ de dados de alturas totais, em que cada variável representou a frequência de indivíduos da i-ésima espécie na j-ésima classe de altura. Essa matriz $\mathrm{X}$ foi o input para as análises de agrupamento e discriminante.

Foi utilizada a distância euclidiana e o método de ligação completa, com o emprego das seguintes fórmulas:

$$
\begin{gathered}
d_{i, j}=\sqrt{\sum_{h=1}^{p}\left(X_{h i}-X_{h j}\right)^{2}}, h=1,2, \ldots, P \\
d_{I, J}=\max _{\substack{i \in I \\
j \in J}} d_{i j}
\end{gathered}
$$

Em que: $d_{i j}=$ distância euclidiana estimada entre as classes $i$ e $j ; X_{h i}=$ número de árvores na classe $i ; X_{h j}=$ número de árvores na classe $j ; d_{I, J}=$ distância entre as médias dos grupos $I$ e $J, h=$ espécies; e $P=$ número total de espécies amostradas.

Após a realização dos agrupamentos, foi realizada uma análise discriminante para confirmar a distinção e classificação dos estratos de altura total obtidos pela análise de agrupamento. Os cálculos relativos a esse método foram realizados por meio do software Statistica ${ }^{\circledR} 7.0$.

\section{Método 3 - IUFRO (LAMPRECHT, 1990)}

A Classificação da IUFRO - International Union of Forest Research Organizations (LAMPRECHT, 1990), proposta por Leibundgut em 1958, estratifica a floresta com base na altura dominante $\left(h_{\text {dom }}\right)$. Assim, a distribuição do número de árvores por classe de altura em estratos é definida da seguinte forma:

Estrato Inferior: $h<h_{d o m} / 3$;

Estrato Médio: $h_{\text {dom }} / 3 \leq h<2 h_{\text {dom }} / 3$;

Estrato Superior: $h \geq 2 h_{\text {dom }} / 3$;

em que: $h=$ altura total e $h_{d o m}=$ altura dominante. 
Método 4 - Calegário et al. (1994)

Essa metodologia de estratificação é feita com base no teste de qui-quadrado. O primeiro passo para se realizar a subdivisão dos indivíduos amostrados em estratos foi ordenar os mesmos crescentemente, tomando-se como base a variável altura. Após a ordenação, utilizando-se o teste qui-quadrado a um nível $\alpha$ de significância, foi verificada a existência ou não de grupos de dados de altura estatisticamente homogêneos, conforme expressão a seguir:

$$
\chi^{2} \text { cal. } i=\left(\frac{S_{i}^{2}}{\bar{H}}\right)(i-1)(i=2,3, \ldots, k, \ldots, n)
$$

em que: $\chi^{2}$ cal. $i=$ Valor do qui-quadrado calculado para i-ésimo grupo de indivíduo; $S_{i}{ }_{i}=$ Variância das alturas dos indivíduos dentro do i-ésimo grupo; e $\bar{H}=$ Média aritmética das alturas dos indivíduos dentro do i-ésimo grupo.

Com esse procedimento, foram obtidos valores de qui-quadrado calculados para diversos grupos de indivíduos. Quando se encontrou um valor que superasse o qui-quadrado tabelado, a $\alpha(5 \%)$ de significância e com i-1 graus de liberdade, concluiu-se que i-ésimo grupo de indivíduos é estatisticamente homogêneo e discriminado como estrato um ou estrato inferior. $\mathrm{O}$ limite inferior desse estrato foi o valor da primeira altura amostrada e o limite superior, o da i-ésima altura.

Após definidos os limites inferior e superior do estrato I, repetiu-se o mesmo procedimento para os indivíduos amostrados com valores de alturas acima do limite superior do primeiro estrato. Com sucessivas estimativas dos valores do qui-quadrado, é possível verificar a existência de outros grupos homogêneos. Haverá tantos estratos quantos forem os grupos homogêneos de indivíduos.

Os cálculos relativos aos Métodos 3 e 4 foram realizados por meio do software Excel $^{\circledR} 2010$.

Os resultados das classificações obtidas para cada método foram comparados à classificação sucessional das espécies, de acordo com os grupos ecológicos a que pertecem, procurando-se definir qual ou quais dos métodos melhor representam a estratificação vertical das florestas estacionais semideciduais.

\section{RESULTADOS E DISCUSSÃO}

Os resultados da estratificação vertical realizada por diferentes métodos propostos para o fragmento florestal em estudo são apresentados a seguir:

\section{Método 1 - Souza (1990)}

Nesse método de estratificação, a floresta é sempre dividida em três estratos de altura total. As divisões estão apresentadas na Tabela 1.

De acordo com esse método, para a comunidade estudada, no estrato I (2,00-6,39 m), a espécie Siparuna guianensis corresponde a $25,9 \%$ do total de indivíduos presentes no estrato. A espécie Guapira opposita vem em seguida com 7,2\%, Apuleia leiocarpa com 6,6\%, Amaioua intermedia com 4,8\% e Myrcia fallax com 3,6\%, do estrato inferior. As espécies que mais ocorrem neste estrato, estão entre as sete de maior VI. Em geral, as espécies que mais se destacam no referido estrato, são classificadas como não pioneiras.

No estrato II (6,40-15,64 m), possuem destaque as espécies: Siparuna guianensis $(12,2 \%)$ e Mabea fistulifera $(10,5 \%)$, que predominam no referido estrato. As espécies Myrcia fallax e Apuleia leiocarpa vem, em seguida, com 4,6 e 3,6\%, respectivamente. Destacam-se também, neste estrato, as espécies Erythroxylum plowmanii (2,9\%) e Guapira opposita (2,7\%). Algumas dessas são pioneiras e outras não pioneiras.

No estrato III (superior), das espécies que atingem mais de 15,65 metros de altura, pode-se citar: Mabea fistulifera (13,6\%), Pseudopiptadenia

TABELA 1: Divisão de estratos verticais, de acordo com o método proposto por Souza (1990), em que: $\mathrm{N}=$ número de indivíduos amostrados; $\mathrm{S}=$ número de espécies amostradas; $\mathrm{F}=$ número de famílias amostradas; e $\mathrm{G}\left(\mathrm{m}^{2} / \mathrm{ha}\right)=$ área basal/ha.

TABLE 1: Vertical stratum division, according to the method proposed by Souza (1990), where $\mathrm{N}=$ number of individuals sampled, $\mathrm{S}=$ number of sampled species, $F=$ number of households sampled, and $\mathrm{G}\left(\mathrm{m}^{2} / \mathrm{ha}\right)=$ area basal $/$ ha.

\begin{tabular}{ccccccc}
\hline Estratos & $\begin{array}{c}\text { Limite dos } \\
\text { estratos (m) }\end{array}$ & $\begin{array}{c}\text { Altura } \\
\text { média } \\
(\mathrm{m})\end{array}$ & $\mathrm{N}$ & $\mathrm{S}$ & $\mathrm{F}$ & $\begin{array}{c}\mathrm{G} \\
\left(\mathrm{m}^{2} / \mathrm{ha}\right)\end{array}$ \\
\hline I & $2,00-6,39$ & 5,31 & 166 & 59 & 25 & 0,58 \\
II & $6,40-15,64$ & 10,14 & 1202 & 211 & 47 & 11,60 \\
III & $15,65-29,20$ & 19,83 & 228 & 90 & 34 & 15,60 \\
\hline Total & & & 1596 & & 27,78 \\
\hline
\end{tabular}


contorta (10,5\%), Apuleia leiocarpa (4,8\%), Virola gardneri (2,2\%) e Piptadenia gonoacantha (2,2\%). Todas essas espécies encontram-se entre as de maior VI. As espécies Byrsonima sericea e Tachigali vulgaris também representam $2,2 \%$ cada, do estrato superior, mas não estão entre as quinze espécies de maior VI. Sendo todas essas, com exceção de Virola gardneri, classificadas como pioneiras, tendo hábito preferencial por ambientes com maior luminosidade para o seu desenvolvimento. Virola gardneri ocorreu apenas uma vez no estrato inferior.

Apuleia leiocarpa, apesar de ser pioneira, ocorreu em destaque no estrato I, porém, obteve maior número de indivíduos nos estratos II e III. A presença dessa espécie no estrato inferior pode estar associada à presença de clareiras. Além disso, ela talvez possua características em se adaptar a ambientes sombreados além de se desenvolver bem em condições de alta luminosidade, podendo ser considerada plástica (que se desenvolve em condições diferenciadas de luminosidade). Assim, mais informações morfológicas e fisiológicas devem ser buscadas para definir a plasticidade da espécie.

No estrato I, houve predominância de Siparuna guianensis e Guapira opposita, sendo as duas não pioneiras. O resultado da divisão do estrato inferior para esse método apresenta algum sentido, quando comparado à classificação ecológica das espécies. Nota-se também que Siparuna guianensis não ocorreu no estrato superior, porém, apareceu com grande frequência nos outros estratos. Guapira opposita ocorreu no estrato superior, mas apenas duas vezes, tendo ocorrido com grande frequência nos menores estratos.

O estrato II (médio) apresenta as espécies Siparuna guianensis, não pioneira e Mabea fistulifera, Myrcia fallax e Apuleia leiocarpa, pioneiras, todas elas distribuídas com grande frequência ao longo deste estrato. Com esse método, não foi possível fazer uma avaliação detalhada do comportamento ecológico das espécies no referido estrato, pois apresenta tendências fortes em concentrar um maior número de indivíduos no estrato médio da floresta, dificultando as interpretações.

Ao se utilizar esse método, interpretações equivocadas sobre o comportamento das espécies nos diferentes estratos podem estar sendo expressas. Esse fato é preocupante, visto que esse método é muito utilizado para a estratificação vertical de florestas, tal como utilizado por Archanjo (2008) e Moreira (2009), em florestas estacionais semideciduais no Espírito Santo; Vilanova (2008), em região de cerrado; Souza (2009), em região de caatinga e Pinto Sobrinho et al. (2009), em floresta estacional semidecidual em Minas Gerais.

Os estratos I e III possuem uma menor concentração de indivíduos, sendo 10,4\% e 14,3\%, respectivamente. De alguma maneira parecem ter sido bem diferenciados, apresentando alguma coerência com a classificação ecológica. De qualquer forma, poderia se obter um melhor resultado se o método possibilitasse a criação de novos estratos.

Com relação à área basal expressa $\mathrm{em} \mathrm{m}^{2} / \mathrm{ha}$, nota-se que ela cresce à medida que se aumenta a altura, ou seja, cresce no sentido dos estratos I, II e III. Apesar de o estrato III possuir um número bem reduzido de indivíduos em relação ao estrato médio, ele possui maior área basal ( $\left.\mathrm{m}^{2} / \mathrm{ha}\right)$, o que se deve ao número de indivíduos com maiores diâmetros no estrato III, fato este comprovado por meio da análise prévia da estrutura diamétrica da floresta que se comporta no formato "J" invertido.

Sabendo-se que a área basal é uma medida de densidade e que estratos com área basal maior ocupam mais o terreno que os estratos com menor área basal, existe uma chance maior do estrato de maior área basal estar sombreando os demais, já que árvores maiores e que expressam maior área basal provavelmente possuem maior copa.

\section{Método 2 - Souza et al. (2003)}

A Figura 1 representa o dendrograma obtido pela análise de agrupamento de classes de altura, apresentando na ordenada valores em porcentagem de dissimilaridade, variando de zero (máxima similaridade) e em torno de 55 (máxima dissimilaridade).

A análise do dendrograma permitiu a indicação da existência de grupos homogêneos e distintos de classes de altura total, confirmando a estratificação vertical em estratos de altura total do povoamento amostrado. O método proposto se mostrou flexível para a estratificação de florestas, porém, não permitiu que esta ocorresse de forma sequencial neste caso.

Assim, pela análise do dendrograma, destacam-se três grupos principais de classes de altura total, convencionados de estratos de altura total (I, II, III), em que as de classes 17 a 29 formam o estrato I, incluindo também árvores pertencentes às classes $2 \mathrm{e}$ 3; as classes de 9 a 16, o estrato II, encontrando-se também neste grupo árvores pertencentes às classes 4 e 5 ; e o estrato III, formado pelas classes 6 a 8 . O estrato I reúne as alturas menos frequentes, isto é, as árvores mais altas ou muito baixas. 


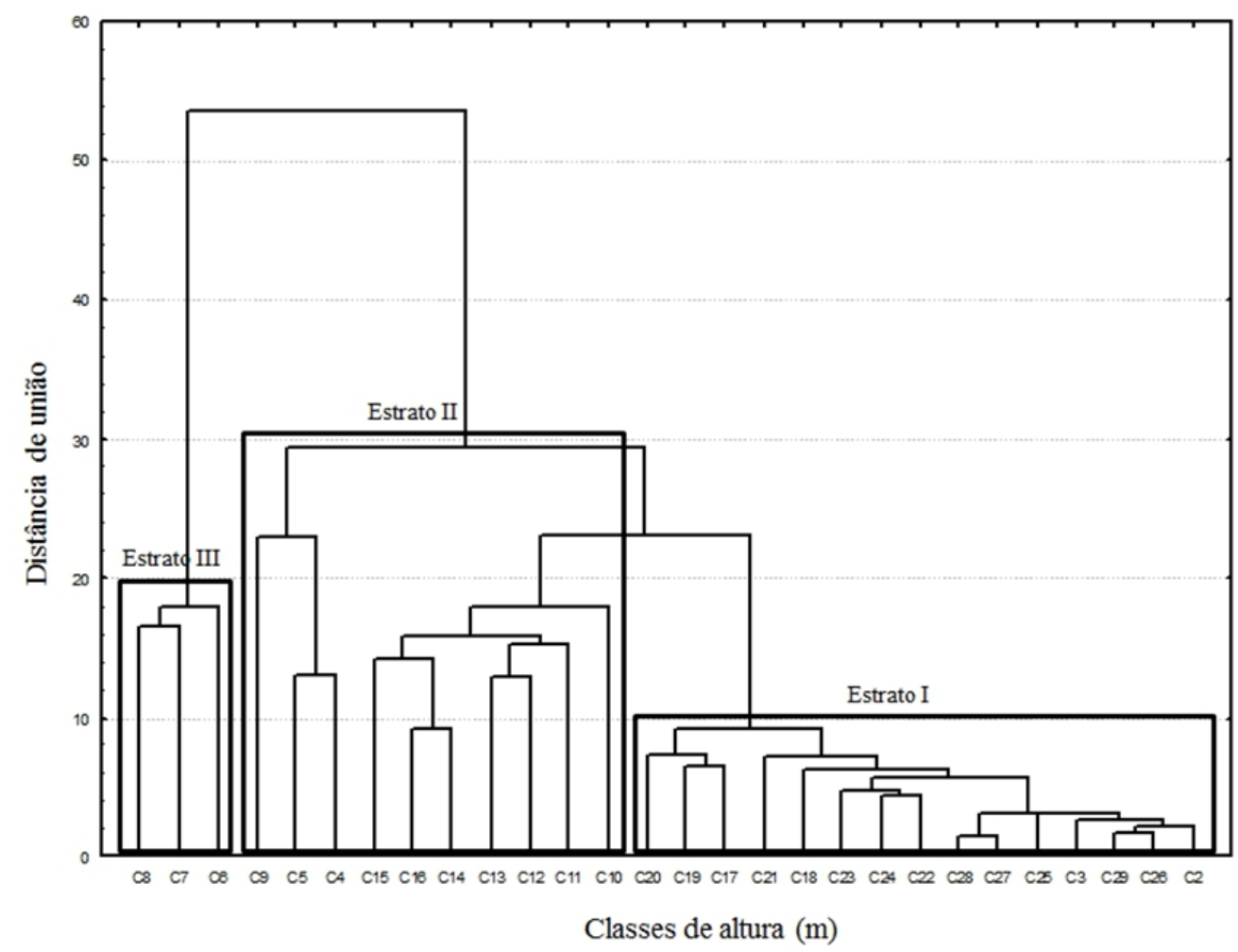

FIGURA 1: Dendrograma de agrupamento das classes de altura, em que as variáveis são as frequências de todas as espécies encontradas na amostragem nas suas respectivas classes de altura.

FIGURE 1: Dendrogram grouping of height classes, in which the variables are the frequencies of all species found in the sample in their respective height classes.

A classificação das árvores individuais em intervalo de $1 \mathrm{~m}$ não permitiu agrupar sequencialmente as classes de altura total (Figura 1). Assim, admitindo-se a apresentação das classes de altura no dendrograma, foi realizado um rearranjo dos grupos, conforme Tabela 2, para que eles se dispusessem de forma sequencial, os estratos que possuiam duas classes independentes de altura total foram desmembrados em a e b.

Após o rearranjo dos estratos, a análise discriminante entre os cinco estratos de altura total obtidos na análise de agrupamento mostrou diferença significativa pelo teste $\mathrm{F}$ e probabilidades de 0,0714 para os estratos I b e II b, de 0,1071 para o estrato III, 0,2857 para o estrato II a e 0,4643, para o estrato I a (Tabela 3 ).

O procedimento final de classificação mostrou que $100 \%$ das classes de altura foram corretamente classificadas nos estratos de altura total (Tabela 3).

Os Métodos 1 e 2 resultaram em um número diferente de estratos, contudo, por meio de uma comparação detalhada, é possível notar a existência de muitas semelhanças.
TABELA 2: Divisão de estratos verticais, de acordo com o método proposto por Souza et al. (2003), em que: $\mathrm{N}=$ número de indivíduos amostrados; $\mathrm{S}=$ número de espécies amostradas; $\mathrm{F}=$ número de famílias amostradas; e $\mathrm{G}\left(\mathrm{m}^{2} / \mathrm{ha}\right)$ = área basal/ha.

TABLE 2: Vertical stratum division, according to the method proposed by Souza et al. (2003), where $\mathrm{N}=$ number of individuals sampled, $\mathrm{S}=$ number of sampled species, $F=$ number of households sampled, and $\mathrm{G}\left(\mathrm{m}^{2} / \mathrm{ha}\right)=$ basal area/ha.

\begin{tabular}{ccccccc}
\hline Estratos & $\begin{array}{c}\text { Limite dos } \\
\text { estratos }(\mathrm{m})\end{array}$ & $\begin{array}{c}\text { Altura } \\
\text { média } \\
(\mathrm{m})\end{array}$ & $\mathrm{N}$ & $\mathrm{S}$ & $\mathrm{F}$ & $\begin{array}{c}\mathrm{G} \\
\left(\mathrm{m}^{2} / \mathrm{ha}\right)\end{array}$ \\
\hline I b & $2,00-3,99$ & 2,98 & 8 & 7 & 5 & 0,04 \\
II b & $4,00-5,99$ & 5,17 & 117 & 46 & 19 & 0,40 \\
III & $6,00-8,99$ & 7,55 & 529 & 129 & 38 & 2,33 \\
II a & $9,00-16,99$ & 12,11 & 760 & 173 & 42 & 10,59 \\
I a & $17,00-29,20$ & 20,71 & 182 & 81 & 32 & 14,42 \\
\hline Total & & & 1596 & & 27,78 \\
\hline
\end{tabular}


TABELA 3: Número de estratos de altura total e porcentagens de classificação usando a análise discriminante TABLE 3: Total height stratum number and percentage of classification using the discriminant analysis.

\begin{tabular}{|c|c|c|c|c|c|c|c|}
\hline \multirow{3}{*}{ Estrato } & \multicolumn{5}{|c|}{ Classificação Prevista } & \multirow{3}{*}{ Total } & \multirow{3}{*}{ Classificação (\%) } \\
\hline & $\mathrm{Ib}$ & II b & III & II a & I a & & \\
\hline & $\mathrm{P}=0,0714$ & $\mathrm{P}=0,0714$ & $\mathrm{P}=0,1071$ & $\mathrm{P}=0,2857$ & $\mathrm{P}=0,4643$ & & \\
\hline $\mathrm{Ib}$ & 2 & 0 & 0 & 0 & 0 & 2 & 100 \\
\hline $\mathrm{IIb}$ & 0 & 2 & 0 & 0 & 0 & 2 & 100 \\
\hline III & 0 & 0 & 3 & 0 & 0 & 3 & 100 \\
\hline IIa & 0 & 0 & 0 & 8 & 0 & 8 & 100 \\
\hline Ia & 0 & 0 & 0 & 0 & 13 & 13 & 100 \\
\hline Total & 2 & 2 & 3 & 8 & 13 & 28 & 100 \\
\hline
\end{tabular}

Ao unir o estrato I b ao II b do Método 2, têm-se como limites inferior e superior as alturas de 2,00 a 5,99 $\mathrm{m}$, sendo que esses dois estratos, apresentam-se muito semelhante ao estrato I do Método 1, que possui como limite superior a altura de $6,39 \mathrm{~m}$.

Ao unir também os estratos III e II a $(6,00$ $16,99 \mathrm{~m}$ ) do Método 2, nota-se a semelhança com o estrato II (6,40-15,64 m) do Método 1. Diferente desse último método, o Método 2 (SOUZA et al., 2003) permitiu um maior nível de detalhamento dos estratos, possibilitando uma discussão mais coerente sobre o comportamento das espécies na floresta.

Assim, as espécies Siparuna guianensis, Mabea fistulifera, Myrcia fallax e Apuleia leiocarpa, que no Método 1 apresentaram-se reunidas com alta frequência no estrato II, no Método 2 puderam ser avaliadas de forma mais detalhada.

Siparuna guianensis ocorria nas alturas mais baixas do estrato II do Método 1, sendo que para este estrato sua altura média foi igual a 7,9 m, já para o presente método (SOUZA et al., 2003), ocorreu com maior intensidade no estrato III $(6,00-$ $8,99 \mathrm{~m})$. No estrato II a (9,00-16,99 m), essa espécie continua a ocorrer, porém, em menor quantidade, pois ao se observar isoladamente, não alcançou altura superior a 13,5 m e em média não ultrapassou $10 \mathrm{~m}$. Com essa avaliação o hábito preferencial dessa espécie por ambientes sombreados é confirmado.

Mabea fistulifera, Myrcia fallax e Apuleia leiocarpa, que são pioneiras e ocorreram em destaque no estrato II do Método 1, concentraram-se nas maiores alturas do estrato, possuindo altura média de $11,1 \mathrm{~m}, 10,7 \mathrm{~m}$ e $10,4 \mathrm{~m}$, respectivamente, mostrando seu destaque nas partes mais superiores do dossel, onde há mais disponibilidade de luz. Porém, no referido método não havia como fazer a distinção do comportamento, já que reunia indivíduos em uma grande amplitude de altura. Já para Método 2, essas espécies ocorreram com maior frequência no estrato II a $(9,00-16,99 \mathrm{~m})$, que possui como limite superior a altura de $16,99 \mathrm{~m}$, englobando, portanto, árvores relativamente altas no dossel e que se desenvolvem bem sob maiores condições de luminosidade. Ainda assim, essas espécies ocorrem no estrato correspondente à altura de 6,00-8,99 m (III), mas em menor número. Dessa forma, é possível notar que o presente método permitiu uma estratificação mais semelhante com a classificação sucessional das espécies.

E finalmente, ao se avaliar o estrato I a do Método 2 e o estrato III do Método 1, nota-se que os limites inferiores de altura foram 17,00 m e 15,65 m, respectivamente, sendo assim, o estrato superior de ambos os métodos foi muito semelhante. E tal como observado no Método 1, o referido estrato apresenta algum sentido ecológico.

$\mathrm{O}$ número de indivíduos nos estratos $\mathrm{I}$, II e III do Método 1 e a união dos estratos a esses correspondentes do Método 2 ficaram assim distribuídos: 166, 1.202 e 228 indivíduos e 125, 1.289 e 182 indivíduos, respectivamente. Apesar dessa diferença entre o número de indivíduos nos estratos correspondentes, os métodos não apresentaram mudanças consideráveis com relação à proporção do número de indivíduos de uma mesma espécie nos correspondentes estratos avaliados.

De acordo com o observado, o Método 2 apresentou a vantagem de permitir que a estratificação fosse realizada com maior nível de detalhamento, quando comparado ao Método 1, de forma que o problema em concentrar um maior número de indivíduos no estrato II foi resolvido.

O método de Souza et al. (2003) estratifica a floresta com base na frequência de indivíduos de cada espécie presente em cada classe de altura, 
sendo que essa particularidade pode ser considerada como uma vantagem do método em relação aos demais, já que eles definem os estratos apenas com base na altura dos indivíduos.

Ao observar a Tabela 2, ordenando os estratos de acordo com o aumento da altura das árvores, ou seja, estratos I b (2,00-3,99 m), II b (4,00$5,99 \mathrm{~m})$, III $(6,00-8,99 \mathrm{~m})$, II a $(9,00-16,99 \mathrm{~m})$ e I a $(17,00-29,20 \mathrm{~m})$, é possível notar que a área basal $\left(\mathrm{m}^{2} / \mathrm{ha}\right)$ aumenta à medida que se aumenta a altura total das árvores. Assim, para o estrato I b, a área basal amostrada foi menor e para o estrato I a foi mais representativa com relação ao total, demonstrando que o estrato I a, apesar de não concentrar o maior número de indivíduos, provavelmente está exercendo a função de sombreador dos demais, já que árvores maiores e que expressam maior área basal provavelmente possuem maior copa.

\section{Método 3 - IUFRO (LAMPRECHT, 1990)}

A altura máxima atingida pelos indivíduos nas parcelas permanentes da amostragem foi de 29,2 m. A partir dessa altura dominante, foram determinados três estratos pelo sistema IUFRO de classificação, conforme a Tabela 4.

Esse método, assim como o Método 1, possibilitou a divisão da floresta em três estratos de al-

TABELA 4: Divisão de estratos verticais, de acordo com o método proposto pela IUFRO (LAMPRECHT, 1990), em que: $\mathrm{N}=$ número de indivíduos amostrados; $\mathrm{S}$ = número de espécies amostradas; $\mathrm{F}$ = número de famílias amostradas; e $\mathrm{G}$ $\left(\mathrm{m}^{2} / \mathrm{ha}\right)=$ área basal $/ \mathrm{ha}$.

TABLE 4: Vertical stratum division, according to the method proposed by IUFRO (LAMPRECHT, 1990), where $\mathrm{N}=$ number of individuals sampled, $\mathrm{S}$ $=$ number of sampled species, $\mathrm{F}=$ number of households sampled, and $\mathrm{G}$ $\left(\mathrm{m}^{2} / \mathrm{ha}\right)=$ basal area $/ \mathrm{ha}$.

\begin{tabular}{ccccccc}
\hline Estratos & $\begin{array}{c}\text { Limite dos } \\
\text { estratos }(\mathrm{m})\end{array}$ & $\begin{array}{c}\text { Altura } \\
\text { média } \\
(\mathrm{m})\end{array}$ & $\mathrm{N}$ & $\mathrm{S}$ & $\mathrm{F}$ & $\begin{array}{c}\mathrm{G} \\
\left(\mathrm{m}^{2} / \mathrm{ha}\right)\end{array}$ \\
\hline I & $2,00-9,72$ & 7,48 & 800 & 168 & 37 & 3,72 \\
II & $9,73-19,47$ & 13,30 & 682 & 167 & 44 & 12,81 \\
III & $19,48-29,20$ & 22,28 & 114 & 56 & 25 & 11,25 \\
\hline Total & & & 1596 & & 50 & 27,78 \\
\hline
\end{tabular}

tura total, contudo, poucos estudos têm adotado essa metodologia para a estratificação.

Nota-se que o primeiro estrato definido $(2,00$ 9,72 m) engloba árvores correspondentes aos três primeiros estratos de altura do Método 2. Sendo assim, se os estratos I b, II b e III do Método 2 têm algum sentido ecológico, conforme já discutido, o estrato I do presente método também possui essa coerência.

$\mathrm{O}$ estrato II do presente método engloba as árvores com altura total de 9,73 a 19,47 m, com a predominância das espécies Mabea fistulifera $(15,25 \%)$, Myrcia fallax (5,13\%) e Apuleia leiocarpa (4,11\%). Em seguida, destaca-se Erythroxylum plowmanii e Unonopsis sp.1, cada uma representando 2,78\% dos indivíduos do estrato II.

Nota-se que Siparuna guianensis não ocupou posição de destaque no estrato II, como esperado, de acordo com a classificação sucessional das espécies, sendo que a maioria dos seus indivíduos $(95,7 \%)$ passou a ocupar o estrato I, fato este justificável para essa espécie de hábito não pioneiro.

Mabea fistulifera, Apuleia leiocarpa e Myrcia fallax, todas pioneiras, destacaram-se em número no estrato II, que apresenta limite superior de 19,47 m, representando bem a classificação ecológica de que estas espécies se desenvolvem bem sob maiores condições de luminosidade.

O que parece é que este método minimizou o problema de divisão encontrado no Método 1, em que a espécie Siparuna guianensis que se encontrava em destaque nos estratos I (2,00-6,39 m) e II $(6,40$ $15,64 \mathrm{~m})$ agora se concentra apenas no estrato I $(2,00$ 9,72 m).

Mabea fistulifera, Apuleia leiocarpa e Myrcia fallax, que se encontravam em destaque no estrato II para o Método 1 (com limites inferior e superior de 6,40-15,64 m respectivamente), juntamente com uma grande concentração de espécies não pioneiras, como a Siparuna guianensis, estão presentes no estrato II do Método 3, mas os limites inferior e superior são maiores (9,73-19,47 m), eliminando a presença de muitas espécies não pioneiras neste estrato, sendo possível uma avaliação ecológica mais coerente.

O estrato III do Método 3 possui como espécies mais representativas Pseudopiptadenia contorta e Mabea fistulifera com 17,54\% e 7,89\% dos indivíduos, respectivamente, destacando-se também Apuleia leiocarpa (4,39\%) e Piptadenia gonoacantha (3,5\%). Assim, o estrato III manteve-se com as mesmas espécies em destaque quando comparado ao Método 1, apesar da grande mudança do limite inferior deste estrato, em que era de $15,83 \mathrm{~m}$ e passou a ter $19,47 \mathrm{~m}$. 
A área basal $\left(\mathrm{m}^{2} / \mathrm{ha}\right)$ foi maior no estrato II, devido ao número de indivíduos com maiores diâmetros com relação aos indivíduos do estrato inferior. Assim, apesar de o estrato I apresentar maior número de indivíduos (800), o valor de área basal foi baixo $\left(3,72 \mathrm{~m}^{2} / \mathrm{ha}\right)$. O estrato III, por sua vez, apresenta indivíduos com maiores diâmetros que os demais, porém, ocorre em pequena quantidade, assim, esse estrato apresentou área basal mais alta que do estrato I, não conseguindo superar a área basal do estrato II, devido ao maior número de indivíduos neste último.

De acordo com a análise da área basal, esse método de estratificação concentrou uma grande quantidade de indivíduos de maior porte no estrato II. Assim, apesar das semelhanças encontradas com a classificação ecológica, esse método não possui embasamento, pois a mudança da altura dominante da amostragem pode mudar toda a discussão. Além disso, esse método leva em conta apenas a altura das árvores, desprezando a relação de frequência, sendo, portanto, insuficiente para uma análise aprofundada do comportamento das espécies nos diferentes estratos da floresta.

\section{Método 4 - Calegário et al. (1994)}

Após a obtenção de valores de qui-quadrado calculados para diversos grupos de indivíduos, concluiu-se a existência de dois grupos homogêneos, sendo, portanto, a floresta em questão dividida em apenas dois estratos de altura estatisticamente distintos entre si, conforme Tabela 5.

Devido à altura máxima amostrada possuir valor relativamente baixo, pelo método proposto não foi possível se chegar ao limite superior do segundo estrato. Portanto, o povoamento amostrado possui indivíduos arbóreos representando apenas um estrato de altura completo, ou seja, estrato I. O número de indivíduos presentes no segundo estrato não foi suficiente para se determinar o limite superior dele.

Sabendo-se que a distinção do estrato II para o Método 1 não foi satisfatória do ponto de vista da classificação ecológica, e que o Método 4 apenas em seu estrato I (2,00-14,60 m), reúne praticamente os limites de altura de dois estratos do Método 1, estrato I (2,00-6,39 m) e II (6,40-15,64 m), pode-se inferir que o Método 4 não trouxe bons resultados para a estratificação da floresta estudada.

Assim, a grande amplitude de limites, caracterizada pela diferenciação tardia dos estratos apenas aos 14,60 metros de altura, e a divisão da floresta em apenas dois estratos não permitiu uma análise detalhada e coerente dos estratos com a classificação sucessional ecológica.

Observando-se a Tabela 5 é possível notar a que a área basal expressa em termos de $\mathrm{m}^{2} / \mathrm{ha}$, é maior no estrato superior, mesmo que concentre um menor número de indivíduos em relação ao outro estrato. Esse resultado era esperado, uma vez que, similarmente à distribuição diamétrica em "J" invertido, característica de floresta inequiânea, as classes superiores de altura total possuem menor número de indivíduos, porém, nas maiores classes de diâmetro.

Os demais métodos de estratificação utilizados neste estudo permitiram que a floresta fosse dividida em um maior número de estratos.

\section{Considerações sobre a estratificação}

$\mathrm{Na}$ área em estudo, algumas parcelas são caracterizadas por perturbações antrópicas, o que poderia estar interferindo na análise dos resultados dos métodos de estratificação. Para avaliar a influência dessas perturbações na disposição dos indivíduos nos estratos verticais, seria necessário um estudo mais aprofundado, considerando a estratificação em cada parcela.

Pela avaliação de cada um dos métodos é possível concluir que a delimitação precisa e distinção dos vários estratos em uma floresta consiste em

TABELA 5: Divisão de estratos verticais, de acordo com o método proposto por Calegário et al. (1994), em que: $\mathrm{N}=$ número de indivíduos amostrados; $\mathrm{S}=$ número de espécies amostradas; $\mathrm{F}=$ número de famílias amostradas; e $\mathrm{G}\left(\mathrm{m}^{2} / \mathrm{ha}\right)=$ área basal/ha.

TABLE 5: Vertical stratum division, according to the method proposed by Calegário et al. (1994), where $\mathrm{N}=$ number of individuals sampled, $\mathrm{S}=$ number of sampled species, $\mathrm{F}=$ number of households sampled, and $\mathrm{G}\left(\mathrm{m}^{2} / \mathrm{ha}\right)=$ basal area/ha.

\begin{tabular}{|c|c|c|c|c|c|c|}
\hline Estratos & $\begin{array}{l}\text { Limite dos } \\
\text { estratos (m) }\end{array}$ & $\begin{array}{l}\text { Altura } \\
\text { média } \\
(\mathrm{m})\end{array}$ & $\mathrm{N}$ & $\mathrm{S}$ & $\mathrm{F}$ & $\begin{array}{c}\mathrm{G} \\
\left(\mathrm{m}^{2} / \mathrm{ha}\right)\end{array}$ \\
\hline I & $2,00-14,60$ & 9,18 & 1282 & 217 & 48 & 9,95 \\
\hline II & $>14,61$ & 18,56 & 314 & 112 & 34 & 17,83 \\
\hline Total & & & 1596 & & & 27,78 \\
\hline
\end{tabular}


uma tarefa difícil, conforme discutido por Sanquetta et al. (2002).

Apesar das coincidências ecológicas que os estratos de alguns métodos possuem, a distinção de vários estratos arbóreos não está claramente estabelecida devido à falta de técnicas que permitam diferenciar os limites precisos de cada estrato, pois na estrutura vertical da floresta, a estratificação e a fisionomia das árvores são difíceis de serem visualizadas através de uma simples tabela de dados. Assim, a elaboração de perfis diagrama tem sido desenvolvida para construir o perfil de uma floresta, adotando-se como modelo a projeção da estrutura espacial sobre um plano vertical.

Longhi (1980) descreveu a estrutura vertical de uma floresta por meio de perfis tridimensionais. Hoje, a descrição espacial de perfis conta com o avanço tecnológico, sendo o Sensor Remoto LIDAR (Light Detectionand Ranging) uma tecnologia de sensoriamento remoto ativo, capaz de fornecer conjuntamente informações horizontais e verticais precisas em alta resolução espacial.

Apesar do potencial promissor da tecnologia LIDAR para uso na área florestal, ela está associada a um alto investimento financeiro, o que é visto como um fator limitante para sua utilização. O desenvolvimento de um estudo espacial em cada parcela do fragmento pode, contudo, alterar toda a classificação obtida pelos métodos utilizados neste trabalho.

\section{CONCLUSÕES}

O método de Souza (1990), que dividiu a floresta em três estratos de altura total, não permitiu uma análise detalhada sobre o comportamento das espécies no estrato II de altura, devido às fortes tendências em concentrar maior número de indivíduos no referido estrato, dificultando as interpretações.

O método de Souza et al. (2003), que leva em consideração a frequência das espécies para a definição de estratos, dividiu a floresta em cinco estratos de altura total, permitindo maior detalhamento dos estratos, possibilitando uma discussão mais coerente sobre o comportamento das espécies na comunidade.

O método da IUFRO dividiu a floresta em três estratos e minimizou o problema em concentrar grande número de indivíduos no estrato II, encontrado no método de Souza (1990), porém, os limites superiores dos estratos I e II foram maiores, concentrando grande quantidade de indivíduos de maior porte no estrato II. Além disso, o método não leva em consideração a frequência de espécies para a definição dos estratos.

O método de Calegário et al. (1994) não trouxe bons resultados para a estratificação, pois ao dividi-la em apenas dois estratos, não permitiu uma análise detalhada e coerente dos estratos com a classificação sucessional em grupos ecológicos obtida.

\section{REFERÊNCIAS BIBLIOGRÁFICAS}

\section{ARCHANJO, K. M. P. A. Análise florística e} fitossociológica de fragmentos florestais de Mata Atlântica no sul do estado do Espírito Santo. 2008. 136 f. Dissertação (Mestrado em Produção Vegetal) - Programa de Pós-Graduação em Produção Vegetal, Universidade Federal do Espírito Santo, Alegre, 2008.

BASSET, Y. et al. Vertical stratification of arthropod assemblages. In: BASSET, Y., NOVOTNY, V.; MILLER, S.E.; KITCHING, R.L.(eds.)Arthropods of tropical forests: spatio-temporal dynamics and resource use in the canopy. Cambridge: Cambridge University Press, 2003. p. 17-27.

CALEGÁRIO, N.; SCOLFORO, J. R. S.; SOUZA, A. L. Estratificação em alturas para floresta natural heterogênea: uma proposta metodológica. Cerne, v. 1, n. 1, p. 58-63, 1994.

CARVALHO, F. A.; NASCIMENTO, M. T.; BRAGA, J. M. A. Estrutura e composição florística do estrato arbóreo de um remanescente de Mata Atlântica Submontana no município de Rio Bonito, RJ, Brasil (Mata Rio Vermelho). Revista Árvore, v. 31, n. 4, p. 717-730, 2007.

CHASE, M. W. An update of the Angiosperm Phylogeny Group classification for the orders and families of flowering plants: APG II. Botanical Journal of the Linnean Society, p. 1-17, 2009.

CIENTEC. Software Mata Nativa 3: Sistema para análise fitossociológica, elaboração de inventários e planos de manejo de florestas nativas. Viçosa, 2010.

DIAS NETO, O.C. et al. Estrutura fitossociológica e grupos ecológicos em fragmento de floresta estacional semidecidual, Uberaba, Minas Gerais, Brasil. Rodriguésia. v. 60, n. 4, p. 1087-1100, 2009. DURIGAN, G. et al. Estrutura e diversidade do componente arbóreo da floresta na Estação Ecológica dos Caetetus, Gália, SP. Revista brasil. Bot., São Paulo, v.23, n.4, p.371-383, 2000.

DURIGAN, G. Estrutura e Diversidade de Comunidades Florestais; Capítulo 6. In: MARTINS 
S.V. Ecologia de Florestas Tropicais do Brasil. Viçosa: Ed. UFV, 2009. p. 261.

FINOL, H. La silvicultura en la Orinoquia Venezolana. Revista Forestal Venezolana. v. 14, n. 25, p. 37-114, 1975.

LAMPRECHT, H. Silvicultura nos trópicos: ecossistemas florestais e respectivas espécies arbóreas. Possibilidades e métodos de aproveitamento sustentado. Eschborn: Deustsche Gessells chaft für technisch Zusammenarbeti (GTZ) GmbH, 1990. 343 p.

LEIBUNDGUT, H. Beispiel einer Bestandesanalyse nach neuen Baumklassen. In: INTERNATIONAL UNION OF FOREST RESEARCH ORGANISATIONS, 12., 1956. Oxford. London. Anais...London. 1958. v. 2. Seção 23 e 24. p. 95118.

LISTA DE ESPÉCIES DA FLORA DO BRASIL. Disponível em: $<$ http://floradobrasil.jbrj.gov. br/2010/>. Acesso em 27 dez. 2010.

LONGHI, S. J. A estrutura de uma floresta natural de Araucaria angustifólia (Bertol.) Kuntze, no sul do Brasil. 1980. 198 f. Dissertação (Mestrado em Ciência Florestal), Universidade Federal do Paraná, Curitiba, 1980.

MOREIRA, L.N. Fitossociologia em ambiente de borda de fragmento de floresta Estacional Semidecidual. Dissertação (Mestrado em Produção Vegetal) - Programa de Pós-Graduação em Produção Vegetal, Universidade Federal do Espírito Santo, Alegre, 2009.

PEREIRA, I. M. et al. Caracterização ecológica de espécies arbóreas ocorrentes em ambientes de mata ciliar, como subsídio à recomposição de áreas alteradas nas cabeceiras do Rio Grande, Minas Gerais, Brasil. Ciência Florestal, v. 20, n. 2, p. 235 253, 2010.

PINTO SOBRINHO, F.A. et al. Composição florística e estrutura de um fragmento de floresta estacional semidecidual aluvial em Viçosa (MG). Floresta, v. 39, n. 4, p. 793-805, 2009.

RODRIGUES, R. R. A sucessão florestal. In: MORELLATO, P. C., LEITÃO FILHO, H. F. (Org.). Ecologia e preservação de uma floresta tropical urbana: Reserva de Santa Genebra. Campinas: UNICAMP, 1995.p. 30-36.

ROLIM S. G. Modelos e Mecanismos de Sucessão Secundária na Floresta Atlântica em Linhares (ES). 2006. Tese (Doutorado em Recursos Florestais - Silvicultura e Manejo Florestal) Escola Superior de Agricultura Luiz de Queiroz, Piracicaba, SP, 2006.
ROLIM, S. G.; COUTO, H. T. Z.; JESUS, R. M. Mortalidade e recrutamento de arvores na Floresta Atlântica em Linhares (ES). Scientia Forestalis, n. 55, p. 49-69, 1999.

SANQUETTA, C. R. Análise da estrutura vertical de florestas através do diagrama h-M. Ciência Florestal, v. 5, n. 1, p. 55-68, 1995.

SANQUETTA, C. R. et al. Estrutura vertical de um fragmento de floresta Ombrófila Mista no Centrosul do Paraná. Floresta. V. 32, n. 2, p. 267-276, 2002.

SANSEVERO, J. B. B.; PIRES, J. P. A.; PEZZOPANE, J. E. M. Caracterização ambiental e enriquecimento da vegetação de áreas em diferentes estágios sucessionais (pasto, borda, clareira e floresta). Revista Científica Eletrônica de Engenharia Florestal . Publicação Científica da Faculdade de Agronomia e Engenharia Florestal de Garça/FAEF. v. 4, n. 07, 2006.

SILVA, A. F. S.et al. Composição florística e grupos ecológicos das espécies de um trecho de Floresta Semidecídua Submontana da Fazenda São Geraldo, Viçosa-MG. Revista Árvore, v. 27, n. 3, p. 311319, 2003.

SOARES, C. P. B., NETO, FRANCISCO de P. ; SOUZA, A. L. de. Dendrometria e Inventário Florestal. Viçosa: Ed. UFV, 2006. 276 p.

SOUZA, A. L. Estrutura, dinâmica e manejo de florestas tropicais. Viçosa: UFV, 1990. 122 p. (Notas de aula).

SOUZA,A.L.; MEIRANETO, J.A.A.; SCHETINO, S. Avaliação florística, fitossociológica e paramétrica de um fragmento de floresta atlântica secundária, município de São Canário, Espírito Santo. Viçosa: Sociedade de Investigações Florestais, 1998. p. 121. (Documento SIF 18)

SOUZA, D. R. et al. Emprego da análise multivariada para estratificação vertical de florestas ineqüiâneas, Viçosa-MG. Revista Árvore, v. 27, n. 1, p. 59-63, 2003.

SOUZA, P. F. Análise da vegetação de um fragmento decaatinga na microbacia hidrográfica do Açude Jatobá - Paraíba. 2009. Monografia (Curso de Engenharia Florestal), Universidade Federal de Campina Grande, Patos, PB, 2009.

VILANOVA, S. R. F. Composição florística e valoração econômica de uma Unidade de Conservação urbana, Cuiabá - Mato Grosso. 2008. Dissertação (Mestrado em Produção Vegetal) - Programa de Pós-Graduação em Ciências Florestais e Ambientais, Universidade Federal de Mato Grosso, Cuiabá, 2008. 